\title{
Atlas maps gene activity, accessibility in developing brain
}

\section{BY ANNA GOSHUA}

30 SEPTEMBER 2021

A new atlas profiles gene expression and the accessibility of DNA in individual cells across the developing human cerebral cortex during mid-gestation, the period when many autism-linked genes are active. The resource could help researchers better understand the molecular underpinnings of cortical development and decipher the effects of rare autism-linked mutations located between genes.

"While we know quite a lot about early stages of brain development, and we know some about middle stages of brain development, mid-gestation and late gestation periods are still to a large extent mysterious," says co-lead investigator Sergiu Pasca, associate professor of psychiatry and behavioral sciences at Stanford University in California. "Our main goal here was to try to capture with single-cell resolution the cell diversity in the transition between states at this key stage of gestation."

To create the atlas, the researchers used 16- to 24-week-old prenatal tissue from the cerebral cortex and sequenced the RNA of 57,868 cells, revealing all active genes in each cell. They also detected regions of chromatin - the coiled complexes of DNA and protein that make up chromosomes - that were accessible to enzymes in 31,304 cells.

Jointly analyzing data from cells in both datasets yielded 185 genes whose expression levels could be predicted from chromatin accessibility patterns: The more accessible an area, the more likely the genes there are expressed. The genes tended to cluster near putative enhancers, or regulatory DNA sequences that are thought to increase gene transcription. This tendency suggests that these genes may help determine the future of immature cells in the developing cortex, the researchers say.

\section{Magnifying glass:}




\section{Spectrum | Autism Research News}

https://www.spectrumnews.org

The researchers used their atlas to investigate the role of spontaneous, or 'de novo,' mutations found between genes in some people with autism. They analyzed more than 200,000 such 'noncoding' mutations found in the whole-genome sequences of 1,902 autistic children or their non-autistic siblings but not their parents. (The data come from the Simons Simplex Collection, which is funded by the Simons Foundation, Spectrum's parent organization.)

They trained a deep-learning model, called BPNet, to predict how the spontaneous mutations disrupt chromatin accessibility profiles for various cell types in the developing brain.

Overall, children with autism have more de novo noncoding mutations than controls, the study shows. These variants frequently disrupted the DNA binding sites of transcription factors such as NRF1, which regulates the expression of a subunit found in some receptors for gammaaminobutyric acid (GABA), a chemical messenger linked with autism and other neuropsychiatric conditions.

"Nobody has ever looked at brain development this way," says Lucia Peixoto, assistant professor of biomedical sciences at Washington State University Spokane, who was not involved with the work.

Prior studies have struggled to establish the significance of noncoding mutations in autism, in part because they did not effectively prioritize areas of the genome that may be relevant for autism. The new atlas enables scientists to focus their analysis on regions implicated in a "process important for autism, like brain development," she says. "It's sort of like having a magnifying glass."

And by linking these mutations to regulatory regions that control gene expression in the developing brain, the atlas enables researchers to assess the functional importance of noncoding mutations. The work appeared in Cell in August.

"In some cases, it's very clear how [mutations are] causing disease, because they're hitting a very important protein, or a very important amino acid in a protein," Pasca says. But when mutations are in noncoding regions, "we're kind of left in the dark as to whether they're having a physiological effect or not. So information about whether those regions where these mutations reside are active, or participating to neural development forces, offers us additional cues in a way about whether they may be involved in cortical development and the pathogenesis of disease."

Moving forward, the researchers plan to use CRISPR engineering to create cell lines that have some of these mutations and to test their effects in cortical organoids - miniature, lab-grown models of the cerebral cortex.

Cite this article: https://doi.org/10.53053/TOKB3054 